\title{
Number Plate Extraction using Template Matching Technique
}

\author{
Pratishtha Gupta \\ Assistant Professor \\ Banasthali University \\ Jaipur, India
}

\author{
G N Purohit \\ Professor \\ Banasthali University \\ Jaipur, India
}

\author{
Manisha Rathore \\ M.Tech Scholar \\ Banasthali University \\ Jaipur, India
}

\begin{abstract}
As an application of CCTV Traffic surveillance, retrieval of the number plate from the vehicles is an important dimension, which demands intelligent solution. In this document, template matching block of computer vision toolbox has been used to extract the vehicle number plate. It is assumed that images of the vehicle have been captured at a particular resolution and orientation. It is also assumed that alphanumeric characters on the plate have been written with a particular font style, type and size.

This paper presents a new SIMULINK model in MATLAB which has been developed to extract the number plate from the vehicle. Each alphanumeric character on a plate is extracted and matched with template image with the help of template matching block. This block matches the pixel by pixel value of original image with one of the template images and gives the template metric value. To develop this SIMULINK model, Digital Signal Processing Toolbox and Computer Vision System Toolbox is used in MATLAB. The approach used for this model is template matching, which has been used for the recognition of letters and digits. This technique can be used for security purpose e.g. finding stolen cars, and for parking area management etc.
\end{abstract}

\section{General Terms}

Digital Signal Processing toolbox, Computer vision System toolbox, Simulink Blockset.

\section{Keywords}

Template matching, MATLAB, vehicle detection, alphanumeric character, CCTV

\section{INTRODUCTION}

Number plate extraction is demanding research area in the field of image processing. An automated system is developed using SIMULINK model in MATLAB which extracts the number plate and recognize alphanumeric characters. Technique used for number plate extraction and recognition is Template matching. Template matching has been done with the help of template matching block of computer vision system toolbox. This block gives the best match of a template within the original image. This block has two input ports; first port is for input image and second is for the template image. Template image should be smaller than the input image. Template matching block gives the best match location of template within the input image and the best matched metric value in output.

In this model each alphanumeric character is extracted from number plate and is matched against templates stored in the database. Each alphanumeric character is matched with all the templates of alphabet and digit. This block matches pixel by pixel value of input image with template image. If all the pixel values are matched it gives template metric value as zero otherwise an integer value is produced.

The paper is organized as follows: Section 2 presents literature survey of number plate extraction and simulink model. Section 3 briefly describes the template matching block. Section 4 presents the proposed methodology for number plate extraction. Section 5 presents the experimental results. Section 6 draws conclusion.

\section{LITERATURE SURVEY}

\subsection{Number Plate Extraction}

Chittode J S et al. [1] proposed an algorithm which is applied on the car park systems to access parking services. Algorithm is based on morphological operations and area criteria test used for number plate recognition. Recognition of characters in number plate is done with optical character.

Peng $\mathrm{H}$ et al. [2] presented an algorithm for "Document Image Recognition". DIR find most matched template for input document image in a database. The algorithm is based on the global matching of CBP.

Chunyu $\mathrm{C}$ et al. [3] presented a methodology which is helpful in recognition of number plate. The methodology was implemented in Matlab. The character is recognized with the help of image pre-processing, edge extraction and segmentation of characters.

Lekhana G.C et al. [4] developed a real time on-line license plate recognition system. LPR algorithm is composed of different steps which are image acquisition, character segmentation through fusion of spectral analysis, character recognition through support vector machines.

Paunwala C.N et al. [5] proposed a method which aims to identify ROI by allowing morphological processing and sequence of directional segmentation. The ROI contains the number plate from which characters are recognized. This method is tested on a database consisting of 750 images at different conditions.

Singh $M$ et al. [6] presented an approach based on morphological opening and closing operations. The number plate is extracted by using this approach. After localization skew correction of number plate is done for effective segmentation of characters. The character extraction is done with the help of template matching.

Kranti S et al. [7] presented a "Feature based number plate localization "method for number plate recognition and extraction. This methodology mainly focuses on two methods i.e. edge detection method and window filtering method. 
Ganapathy V et al. [8] proposed a methodology which is helpful in the number plate recognition for vehicles in Malaysia. The proposed methodology is the combination of morphological process and Hough transform. An experimental result shows accuracy of $95 \%$.

Othman K et al. [9] used texture based approach which is based on edge information for the license plate localization and recognition. Segmentation of characters is done with the help of multi layer perceptron neural network.

\subsection{Simulink Model Blockset}

Gupta P et al. [10] developed a Simulink model for the traffic load computation. The approach used is corner detection. This block counts the number of corners of vehicle and with the help of corners number of vehicles is calculated at the junction.

Kamboj A et al. [11] presented an efficient Simulink model for image segmentation. The main focus is on processing an image pixel by pixel and modification of pixel of neighborhoods. Blocks of video and image processing are used.

Alalfi $\mathrm{M} \mathrm{H}$ et al. [12] described code-based clone detection technique for efficient identification of near miss clone in Simulink models. Main aim to leverage text based clone detection technique.

Boulmalf $M$ et al. [13] provided an efficient method for teaching digital and analog modulation for undergraduate students in an information technology program. Simulink model simulates analog and digital modulation techniques exempting any derivation of mathematical formulas.

\section{TEMPLATE MATCHING BLOCK}

Template matching block is used for matching the template image within input image. The template image and the input image are of same orientation and resolution. Size of template image should be smaller than the input image. Template matching block belongs to Computer Vision System Toolbox, from analysis and enhancement library. Port one is used to input the input image and port two is used to input the template image. Output port gives the output as best match location as well as template metric value. Block looks like as own in figure1.

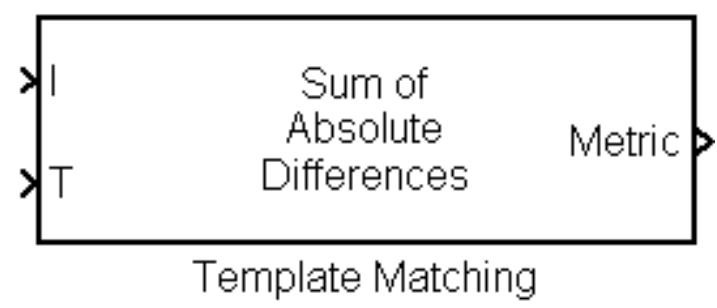

Fig 1. Template Matching Block

The template metric value can be calculated as sum of absolute differences, sum of squared differences or maximum absolute differences.

\subsection{Sum of Absolute Differences}

Metric value calculated by SAD is known as Taxicab distance metric. SAD value estimates the best position of template within the input image. It is calculated as:

$$
d_{1}\left(I_{j}, T\right)=\sum_{i=1}^{n}\left|I_{i . j}-T_{i}\right|
$$

\subsection{Sum of Squared Differences}

The metric value calculated by SSD is known as Euclidean Distance metric. It also estimates the best match position of template within input image. It is calculated as:

$$
d_{2}\left(I_{j}, T\right)=\sum_{i=1}^{n}\left|I_{i . j}-T_{i}\right|^{2}
$$

\subsection{Maximum Absolute Differences}

The metric calculated by MAD is uniform distance metric. It also estimates the best match position of template. It is calculated as:

$$
d_{\infty}\left(I_{j}, T\right)=\lim _{x \rightarrow \infty} \sum_{i=1}^{n}\left|I_{i . j}-T_{i}\right|^{p}
$$

\section{PROPOSED METHODOLOGY}

The methodology is shown in the flowchart. The basic approach of our model is template matching. This approach is helpful in character recognition after extraction of alphanumeric characters. Number Plate Extraction flowchart is shown in figure 2 .

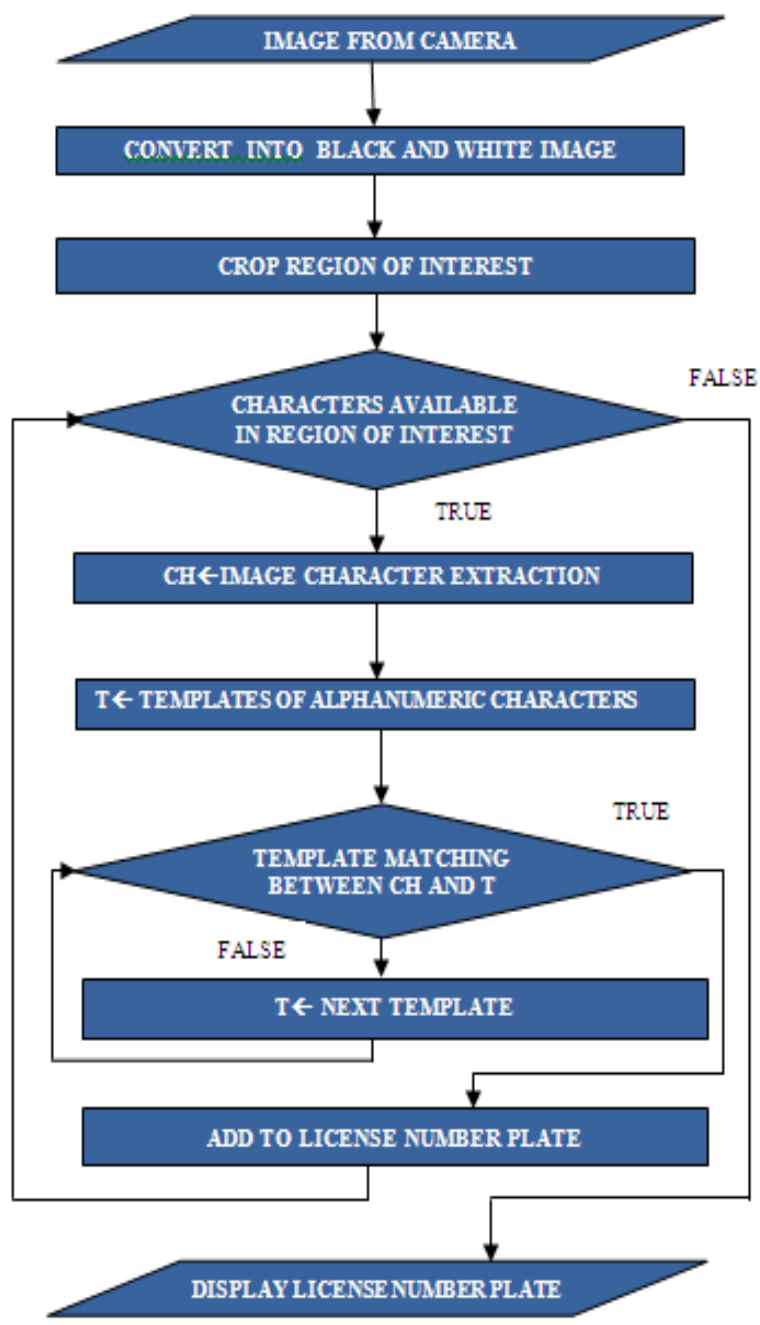

Fig 2. Flowchart for Number Plate Extraction

Blocks shown in table 1 are used to build the Simulink model: 
Table 1. Number of Blocks

\begin{tabular}{|c|c|c|}
\hline Block & Library & Quantity \\
\hline $\begin{array}{c}\text { Image From } \\
\text { File }\end{array}$ & $\begin{array}{c}\text { Computer vision } \\
\text { System } \\
\text { Toolbox/Sources }\end{array}$ & 8 \\
\hline Selector & $\begin{array}{c}\text { DSP system toolbox/ } \\
\text { Signal management/ } \\
\text { Indexing }\end{array}$ & 7 \\
\hline Template & $\begin{array}{c}\text { Computer Vision } \\
\text { System Toolbox/ } \\
\text { Analysis and } \\
\text { enhancement }\end{array}$ & 7 \\
\hline Display & $\begin{array}{c}\text { Signal Processing } \\
\text { Blockset/Signal } \\
\text { Processing /Sinks }\end{array}$ & 7 \\
\hline
\end{tabular}

Following steps are used to build simulink model for number plate extraction:

1. Image is converted into black and white image (using adobe Photoshop).

2. Image from file block (figure 3 and figure 4 ) is used to load image.

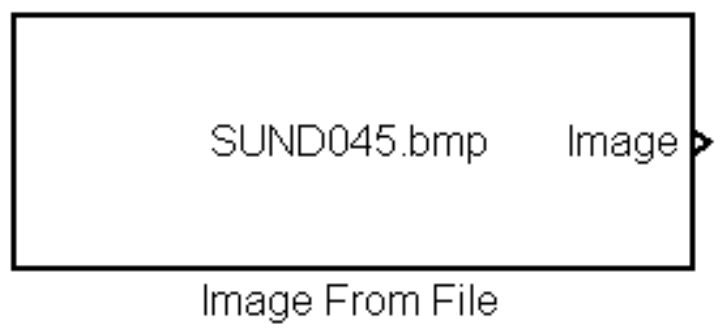

Fig 3. Image From File Block

Main Data Types
Farameters
File name: AphotoshopiSUND045.bmp Browse...
Sample time: inf
Image signal: One multidimensional signal

Fig 4. Source Block Parameter

Input image is shown in figure 5, from which alphanumeric characters are recognized.

\section{S UN D 045}

Fig 5. Input Image

3. From input image individual characters are extracted with the help of selector block..

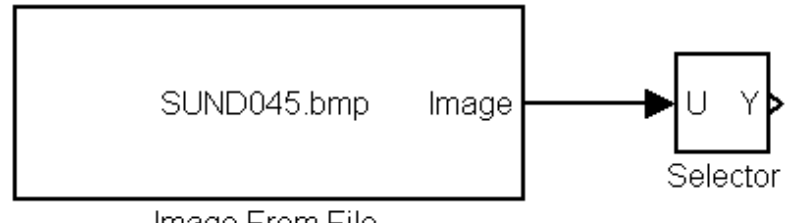

Image From File

Fig 6. Selector Block

\begin{tabular}{l} 
Number of input dimensions: \\
Index mode: One-based \\
\begin{tabular}{|l|l|r|r|r|}
\hline & Index \\
1 & Starting index (dialoq) & Option & Index & Output Size \\
\hline 2 & Starting index (dialog) & $\checkmark$ & 1 & 100 \\
\hline
\end{tabular} \\
Sample time (-1 for inherited): -1 \\
\hline
\end{tabular}

Fig 7. Source Block Parameter

4. Template image is loaded with the help of image from file block (figure 8).Template image is loaded in similar manner as in step1.
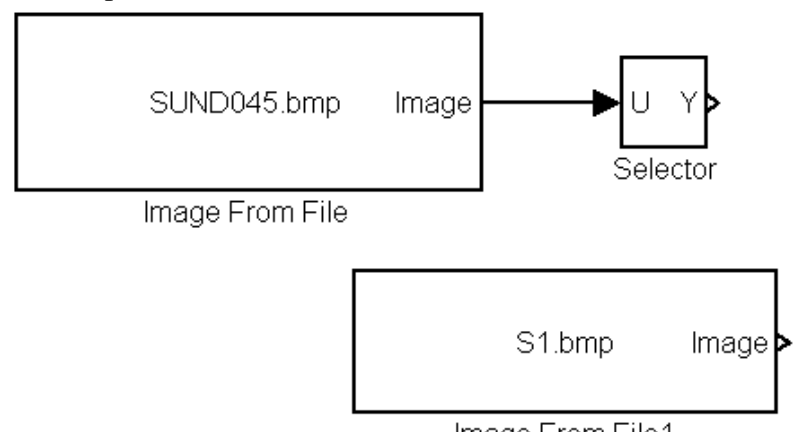

Image From File1

Fig 8. Model at step 3.

5. Template matching block is used to match the template within the input image (figure 9). Some properties of block are set as shown in figure 10 .

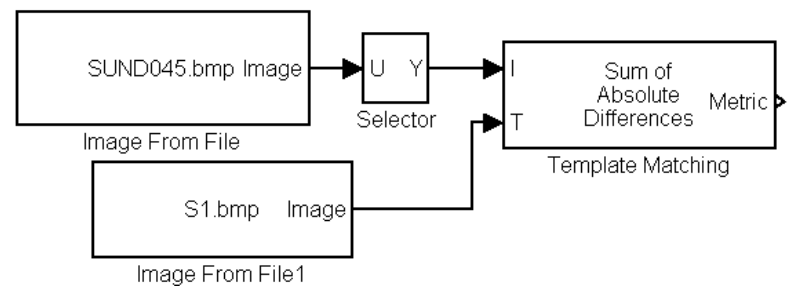

Fig 9. Model at step 4.

Main Data Types
Parameters
Match metric: Sum of absolute differences
Output options
Output: Metric matrix

Fig 10. Source Block Parameter

6. If the output of template metric value is zero, it means the template is matched within input image (figure 11). 


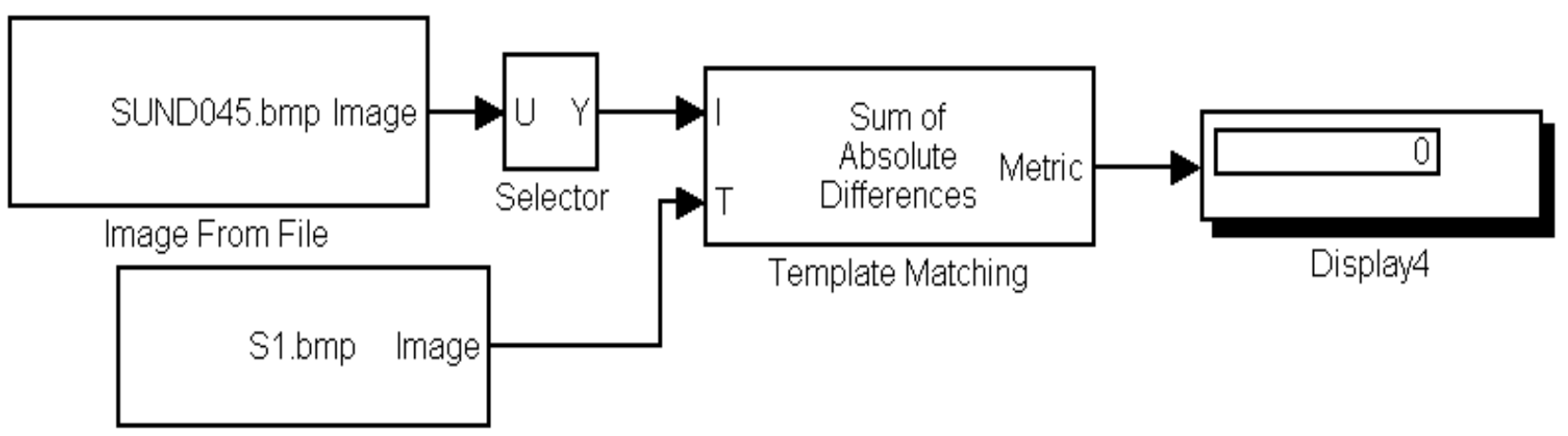

Image From File1

Fig 11. Model for Single Character

Simulink model for full number plate extraction and Recognition is as shown in figure 12 .

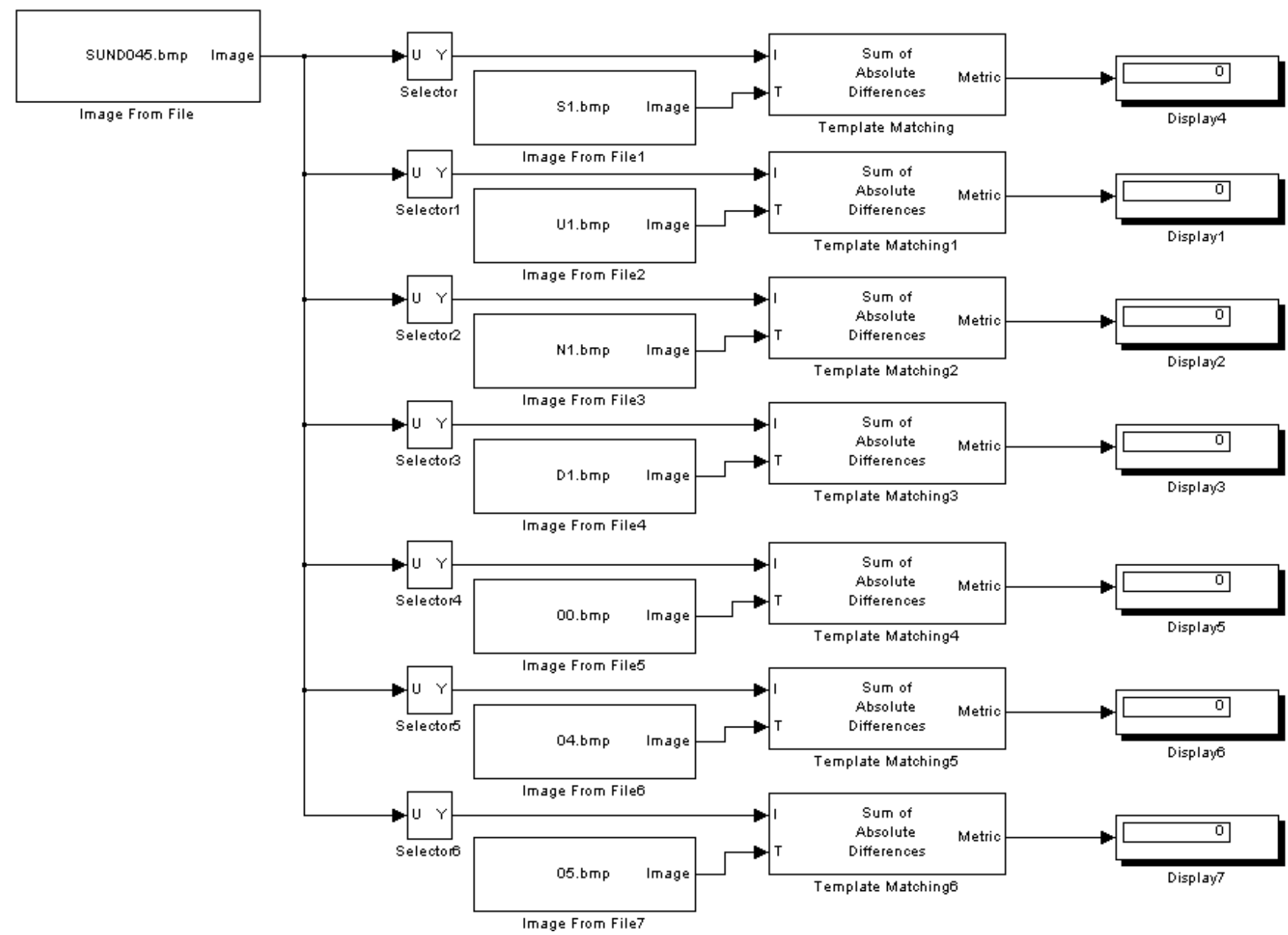

Fig 12. Final Model For Number Plate

To run this Simulink model in Matlab environment Configuration parameter are set. Configuration parameter dialog box is opened by selecting simulation menu from model. Parameters are set as follows:

Solver pane, start time $=0$, stop time $=0$
- Diagnostics pane, Automatic solver parameter selection $=$ set as none

Template images are stored in database for character recognition (figure 13):

- $\quad$ Solver pane, type= variable step, solver $=$ ode 45 


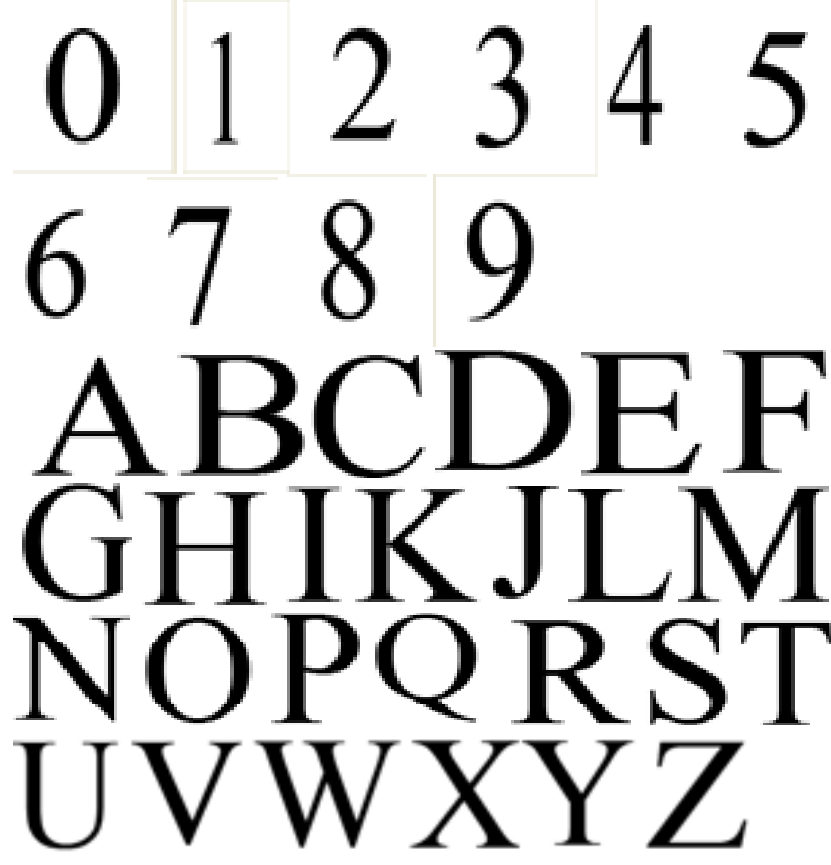

Fig 13. Template Images

\section{RESULT}

Result of model shows, alphanumeric character are extracted and recognized properly. It gives accurate result for alphanumeric characters which have been written with a similar font style, type and size with same resolution.

Table 2. Result

\begin{tabular}{|l|l|l|l|}
\hline Techniques & Real Images & $\begin{array}{l}\text { Number } \\
\text { Plate } \\
\text { extraction }\end{array}$ & $\begin{array}{l}\text { Result } \\
\text { Accuracy }\end{array}$ \\
\hline $\begin{array}{l}\text { Template } \\
\text { Matching }\end{array}$ & 10 & 10 & $100 \%$ \\
\hline
\end{tabular}

\section{CONCLUSION}

We conclude from analysis that number plate is properly extracted and recognized with $100 \%$ accuracy. Model has been developed using Simulink Blockset in Matlab which can be implemented on the hardware by using the HDL code generator in Matlab and real time number plate extraction system is made.

\section{FUTURE SCOPE}

There is a scope in the future where the system can be able to work where the number plate, the color and the font of the plate is identical with varied font sizes. The system should not compromise and it should be sensitive and should be able to locate the plate at any conditions as tracking stolen vehicles and monitoring vehicles for homeland security.

\section{REFERENCES}

[1] J.S. Chittode and R. Kate, "Number plate recognition using segmentation," International Journal of Engineering Research \& Technology, Vol. 1 Issue 9, November- 2012

[2] H. Peng, F. Long and Z. Chi, "Document image recognition based on template matching of component block projections," IEEE transaction on Pattern Analysis and machine Intelligence, Vol. 25, no. 9, pp 1188-1192, sep 2003.

[3] C. Chunyu, W. Fucheng, C. Baozhi and Z. Chen," Application of image processing to the vehicle license plate recognition," International Conference on Computer Science and Electronics Engineering, published by Allantis press, pp 2867-2869, 2013.

[4] G. C.Lekhana and R.Srikantaswamy," Real time license plate recognition system," International Journal of Advanced Technology \& Engineering Research, Vol-2, Issue-4, pp 5-9, July 2012.

[5] C N Paunwala and S Patnaik, "A novel multiple license plate extraction technique for complex background in Indian traffic conditions," International Journal of Image processing, Vol-4,Issue-2,pp 106-118.

[6] P Pandya and M Sing," Morphology based approach to recognize number plates in India," International Journal of Soft Computing and Engineering,Vol-1, Issue-3, pp 107-113, June2011.

[7] S Kranti and K Pranathi," Automatic number plate recognition," International Journal of Advancements in Technology,vol-2, no-3, pp408-423, July 2011.

[8] V Ganapathy and W.L.D Lui," A Malaysian vehicle license plate localization and recognition system," Journal of Systemic and Cybernetics (pdf from freewebs.com)

[9] O Khalifa, S Khan, R Islam and A Suleiman," Malaysian vehicle license plate recognition," The International Arab Journal of Information Technology, vol-4, no-4, pp 359365, Oct 2007.

[10] P Gupta, G. N. Purohit and A. Gupta," Traffic load computation using corner detection technique in Matlab Simulink model," International journal of computer application,Vol-72, no-16, 2013.

[11] Akamboj and A Gupta," Simulink model based image segmentation," International Journal of Advanced Research in Computer Science and Software Engineering, vol-2, no-6, pp 146-150, June 2012.

[12] M H Alalfi, J R Cordy, T R Dean, M Stephen and A Stevenson," Near-miss model clone detection for Simulink model. 2012 ieeexplore.

[13] M Boulmalf, Y Semmar, A Lakas and K Shuaib," Teaching digital and analog modulation to undergraduate information technology students using Matlab and Simulink, "IEEE EDUCON education engineering, pp 685-692, April 2010. 